\title{
COMPETITION BETWEEN INTRAMOLECULAR VIBRATIONAL ENERGY RE-DISTRIBUTION AND UNIMOLECULAR DISSOCIATION: A SCATTERING THEORETICAL POINT OF VIEW
}

\author{
KIYOHIKO SOMEDA and HIROKI NAKAMURA \\ Division of Theoretical Studies, Institute for Molecular Science, Myodaiji, \\ Okazaki 444, Japan \\ FREDERICK H. MIES \\ Molecular Physics Division, National Institute of Standards and Technology, \\ Gaithersburg, Maryland 20899, U.S.A.
}

(Received 15 March, 1994)

\begin{abstract}
Systematic behavior of decay rates of resonances above dissociation threshold is investigated by using the theory of resonance scattering. The condition for the Rice-Ramsperger-Kassel-Marcus (RRKM) rate formula to be valid is clarified by analyzing the random model of unimolecular dissociation. The decay rate averaged over many resonances agrees with the RRKM rate when the mean spacing and the mean width of the resonance states coincide with each other. On the other hand, auto- and mutual-correlation functions of the non-stationary wave functions indicate a rather paradoxical and intriguing phenomenon: In the RRKM regime, insufficient time is left for intramolecular vibrational energy redistribution (IVR) before dissociation.
\end{abstract}

KEY WORDS: Unimolecular dissociation, RRKM theory, IVR

\section{INTRODUCTION}

The statistical theories of chemical reaction, ${ }^{1}$ such as the transition state theory ${ }^{2}$ and the phase space theory, ${ }^{3,4}$ have been discussed in connection with the so called "chaotic" behavior ${ }^{5}$ in reaction systems. In particular, the Rice-Ramsperger-KasselMarcus (RRKM) theory ${ }^{6,7}$ of unimolecular dissociation has been believed to have its ground in the "chaotic" dynamics of highly excited molecules. According to the most naive interpretation, for instance, the RRKM theory is based on the "chemical equilibrium" between the excited states and the "transition state". The RRKM rate formula is given by 


$$
k_{\mathrm{RRKM}}=\frac{1}{2 \pi \hbar} \frac{K^{\ddagger}}{\rho}
$$

which depends only on the density of states $\rho$ of the excited molecule and the number of open channels $K^{\ddagger}$ at the transition state. Since the RRKM theory assumes complete loss of state-selectivity, the RRKM rate formula has been believed to be valid when intramolecular vibrational energy re-distribution (IVR) is so fast and complete that the dissociation process is independent of the initial state or ensemble.

In recent years, decay rate of single rovibrational state among the manifold located above dissociation threshold has been observed, ${ }^{8-10}$ and the state-specificity of the decay rate is found to remain even in such a system that is believed to be statistical. The observed state-dependency is, however, very complex and can be regarded as a "fluctuation" rather than "selectivity". The decay rate averaged over many states distributed in a narrow energy range has been shown to agree with the RRKM rate in which the barrier penetration is taken into account. This experimental observation confirms the new point of view that the RRKM rate formula should be understood as the one to provide the average decay rate..$^{8,9}$ On the other hand, the "fluctuation" of decay rate has also been investigated ${ }^{11-13}$ under the strong influence of the random matrix theory, ${ }^{14}$ which has been intensively discussed in the field of quantum chaos. A spectroscopic analysis has indicated that the molecular Hamiltonian matrix elements relevant to the observed spectrum can be regarded as Gaussian random numbers. ${ }^{8,9}$

When unimolecular dissociation is discussed in relation with the RRKM theory, usually it is not a direct dissociation, but a vibrational predissociation. The latter is nothing but a decay of resonance above dissociation threshold, and can be described by the theory of resonance scattering. ${ }^{15}$ The authors have studied the nature of the average decay rate by using a random matrix model of unimolecular dissociation ${ }^{16,17}$ based on the quantum theory of resonance scattering. Special attention has been paid to the correlation among the average resonance width, the density of resonance states and the magnitude of coupling connecting the resonance states with continua. The random model has revealed a clear connection between the overlapping resonance and the RRKM rate: The average decay rate agrees with the RRKM rate when the mean spacing of resonances coincides with their average resonance width. The fluctuation of the decay rates is found to remain even when the average decay rate agrees with the RRKM rate.

In the present paper, we focus our attention to the connection between the intramolecular dynamics and the RRKM theory. It is generally believed that in the RRKM regime state-selectivity is completely lost, i.e., decay rate should be equal for all states, because very rapid and complete IVR occurs before dissociation. In order to examine the competition between IVR and dissociation, auto- and mutualcorrelation functions of non-stationary wave functions of the excited molecule are calculated based on the random model of unimolecular dissociation. The obtained results indicate an intriguing paradoxical phenomenon: No sufficient time is left, particularly in the RRKM regime, for IVR to be completed before dissociation. 
The present paper is organized as follows: In section 2, the theory of resonance scattering is briefly surveyed. The basic framework and results of the random model, which has already been reported in refs. 16 and 17, are summarized in section 3 . The auto- and mutual-correlation functions generated by the random model are discussed in section 4 . Section 5 summarizes the results.

\section{FESHBACH THEORY OF RESONANCE SCATTERING}

In this section, the essence of the Feshbach theory of resonance scattering ${ }^{18-20}$ is briefly surveyed. Suppose that $N$ zero-th order resonance states, $|n\rangle(n=1, \ldots, N)$, are interacting with $K$ continua, $|k E\rangle(k=1, \ldots K)$, as shown in fig. 1 , where $k$ represents the index of continuum and $E$ represents energy. The coupling between $|n\rangle$ and $|k E\rangle$ causes the decay of the resonance state. The coupling matrix element is denoted as

$$
V_{n k}=\langle n|H| k E\rangle,
$$

where the $E$-dependence of this matrix element is not essential to discuss the qualitative physics of resonance and is neglected. ${ }^{20}$ The positions and widths of the resonances can be obtained from the eigenvalues of the non-hermitian matrix,

$$
\mathbf{H}_{\text {eff }}=\mathbf{E}_{Q}-i \pi \mathbf{V}{ }^{t} \mathbf{V} \text {, }
$$

where $\left(\mathbf{E}_{Q}\right)_{n m}=\langle n|H| m\rangle$ and $t$ represents the transpose of the matrix. The eigenvalues of $\mathbf{H}_{\text {eff }}$ can be written as $\Lambda_{v}=E_{v}-i \Gamma_{v} / 2$, from which the position $E_{v}$ and the width $\Gamma_{v}$ of the $v$-th resonance are obtained.

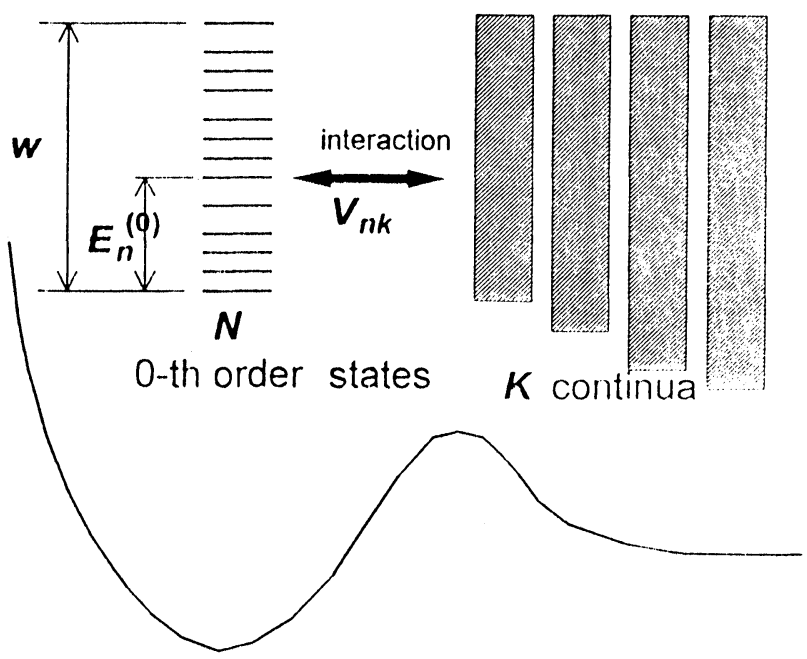

Figure 1: A schemetic illustration of the unimolecular dissociation as the decay of resonance states located above dissociation threshold. In the random model, $N$ zero-th order resonance states are distributed in the energy interval $w$. Each zero-th order resonance states interact with the $K$ continua through the coupling matrix element $V_{n k}$. 


\section{RANDOM MODEL OF UNIMOLECULAR DISSOCIATION AND THE RRKM RATE}

When the resonance widths $\Gamma$ are smaller than their spacing $1 / \rho$, the resonances are observed as isolated sharp peaks in an absorption spectrum. In such case, the decay rate $k_{\mathrm{d}}$ satisfies the relation

$$
k_{d}=\frac{\Gamma}{\hbar}<\frac{1}{\hbar \rho}
$$

Usually the number of open channels are very large, and the inequality $K^{\ddagger}>2 \pi$ holds except for the dissociation very close to the threshold. In this case, inequality (4) leads to $k_{\mathrm{d}}<k_{\mathrm{RRKM}}$, i.e. the decay rate is always smaller than the RRKM rate if the resonances are isolated. This implies that the decay rate agrees with the RRKM rate when the resonances overlap with each other. ${ }^{6}$ In the theory of atomic collision it is well known that spectral profile of resonances changes systematically when the resonances overlap with each other. ${ }^{21-23}$ Such nature of resonance scattering has been shown to be connected with the RRKM theory by analyzing the random model as follows:

In order to understand the nature of resonance scattering particularly under the regime of overlapping resonance, the following "random model" has been devised. The details of the random model has already been reported in refs. 16 and 17 . The essence of the model is to use random numbers for constructing the matrix $\mathbf{H}_{\text {eff }}$ of eq. (3). A proper choice of the basis makes $\mathbf{E}_{\mathrm{Q}}$ diagonal. The diagonal matrix elements of $\mathbf{E}_{\mathrm{Q}}$, which are zero-th order energy positions of resonances, are generated by uniform random numbers distributed in the interval $[0, w]$. On the other hand, the interaction matrix elements $V_{\mathrm{nk}}$ are generated by random numbers obeying the Gaussian distribution with zero average and the standard deviation v, i.e., $N(0, v)$. As mentioned in section 1, there is an evidence that the interaction matrix elements can be regarded as Gaussian random numbers. ${ }^{89}$ The mean magnitude of the coupling is represented by $v$. The parameters of this random model are summarized as follows: the number of zero-th order resonance states $N$, the number of continua $K$, the energy interval $w$ in which the zero-th order resonance states are distributed, and the mean magnitude of the coupling matrix elements $v$. The density of states $\rho$ is given by $\rho=N / w$. By changing these four parameters, the eigenvalues of the matrix given in eq. (3), and thus the resonance width $\Gamma_{v}$ are numerically obtained. The results are briefly summarized below.

As the density of state $\rho$ increases, the overlap of resonance occurs at the critical density $\rho_{\mathrm{c}}=(\pi \mathrm{v})^{-2}$. In the overlapping regime $\rho>\rho_{\mathrm{c}}$, the distribution of resonance width is bifurcated into two branches, the short- and long-lived branches (see fig. 2). The resonance states of the short-lived branch have very short lifetimes and cannot be recognized as resonance states. In practice, they have character of continuum and are relevant not to the vibrational predissociation of the present interest but to direct dissociation. The average resonance width $\langle\Gamma\rangle$ is meant here by the average of resonance widths of the long-lived branch. The average resonance width exhibits systematic behavior as a function of the parameters $K, \rho$ and $v$. It can be predicted by the formula originally derived in the picket fence model, ${ }^{15}$ 


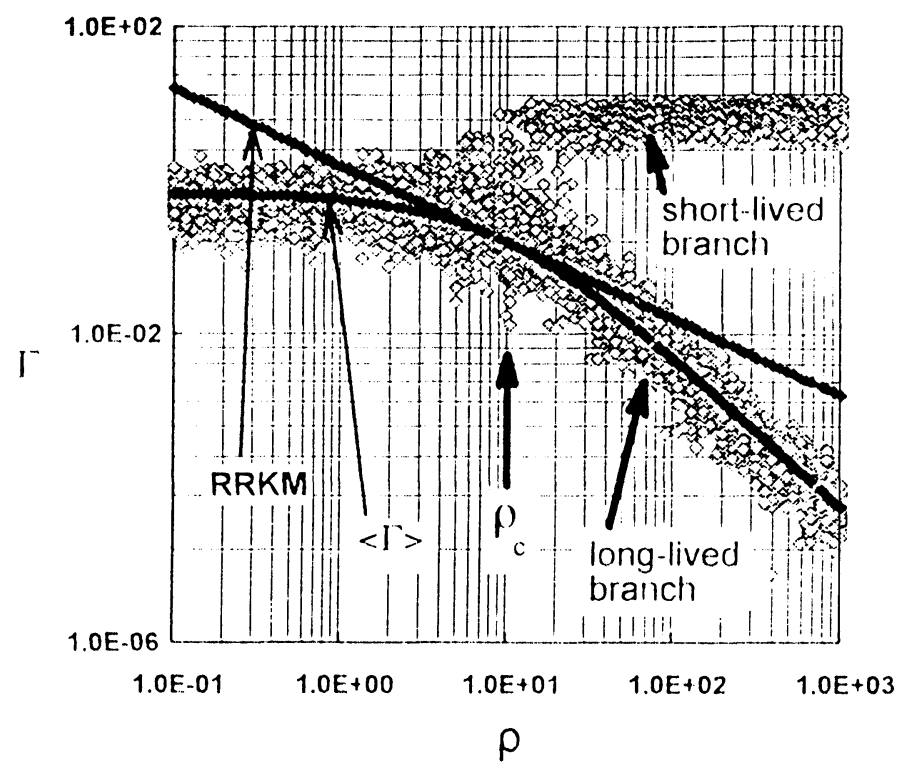

Figure 2: The systematic behavior of the resonance widths $\Gamma$ in the random model as a function of the density of resonance states $\rho$. The parameters are $N=100, K=10$ and $v=0.1$. At the critical density $\rho_{\mathrm{c}}$ (in this case $\rho_{\mathrm{c}}=10.1$ ), where the resonances begin to overlap with each other, and the distribution of $\Gamma$ bifurcate into the short- and long-lived branches. The states belonging to the short-lived branch are essentially the continuum states and not resonances in practice. The curve represents the formula given in eq. (5) describing the overall behavior of average resonance width. The resonance width derived from the RRKM rate is also shown. It can be seen that the average resonance width agrees with the RRKM estimation at the critical overlap.

$$
\langle\Gamma\rangle=\frac{2 K \pi v^{2}}{\left(1+\pi^{2} v^{2} \rho\right)^{2}}=\hbar k_{\mathrm{RRKM}}\left(\frac{\sqrt{\rho / \rho_{c}}+\sqrt{\rho_{d} \rho}}{2}\right)^{-2}
$$

The average decay rate $\langle\Gamma\rangle / h$ is smaller than the RRKM rate except at the critical overlap, $\rho=\rho_{\mathrm{c}}$, where $\langle\Gamma\rangle / h$ agrees with the RRKM rate (see fig. 2).

Absorption spectra to the manifold of the resonance states can be also generated in the random model. The absorption intensity is given by ${ }^{24,25}$

$$
I(E) \propto-\operatorname{Im} \sum_{n^{\prime}} \sum_{n} \sum_{\nu}\left\langle\Phi_{\mathrm{i}} \mid n^{\prime}\right\rangle\left\langle n^{\prime} \mid v\right\rangle \frac{1}{E-\Lambda_{\nu}}\langle v \mid n\rangle\left\langle n \mid \Phi_{\mathrm{i}}\right\rangle,
$$

where $\left|\Phi_{\mathrm{i}}\right\rangle$ is the initial state of the absorption, $\langle v|$ and $|v\rangle$ are the eigen-bra and ket vectors of $\mathbf{H}_{\text {eff }}$, respectively. The Franck-Condon factor $\left\langle\Phi_{i} \mid n\right\rangle$ is generated again by Gaussian random numbers. The spectrum is found to be composed of sharp isolated peaks in the isolated regime, $\rho<\rho_{\mathrm{c}}$, and also in the overlapping regime, $\rho>$ $\rho_{\mathrm{c}}$, while at the critical overlap, $\rho=\rho_{\mathrm{c}}$, the spectral profile is so diffuse that peaks are not distinguished from each other as can be seen in fig. 3. It should be noted that the overlapping regime does not mean real overlap of resonances. In this regime new states are formed by linear combinations of the original zero-th order states, 


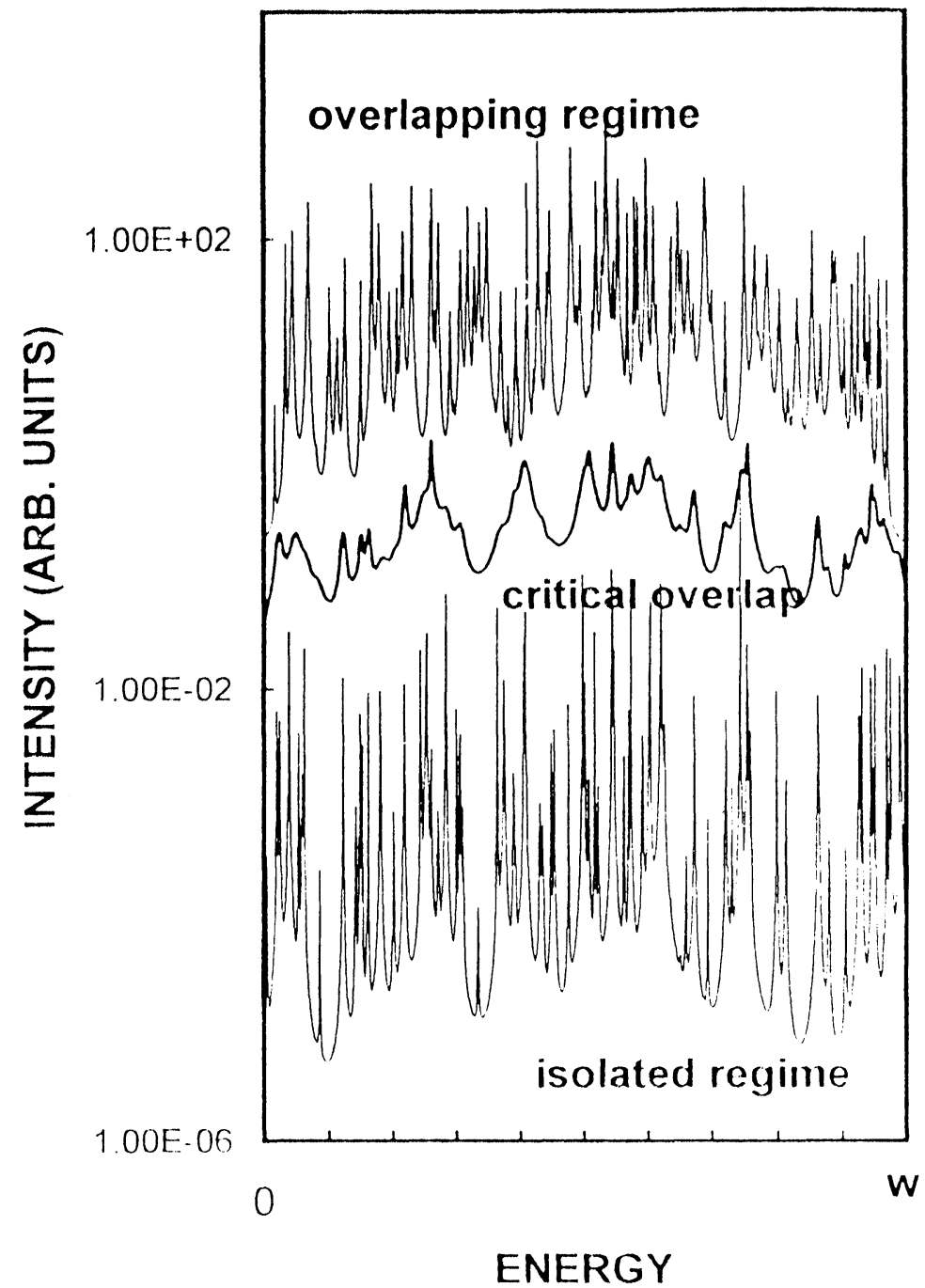

Figure 3: The absorption spectra generated in the random model according to eq. (6). The parameters are $N=100, K=10$ and $v=0.1$. The vertical scale is logarithmic, while the horizontal scale is linear. The bottom spectrum corresponds to the regime of isolated resonance with $\rho=\rho_{\mathrm{c}} / 100$, the middle thick spectrum to the critical overlap with $\rho=\rho_{\mathrm{c}}$, and the top spectrum to the overlapping regime with $\rho=$ $100 \rho_{\mathrm{c}}$. The spectrum is most diffuse in the case of critical overlap.

and they form shifted isolated resonances. The real overlap is attained at the critical overlap.

The reason why the RRKM formula becomes valid at the critical overlap can be explained as follows: The RRKM theory assumes that the decay rate is given by the outgoing flux at the transition state. ${ }^{26-28}$ It has been shown that the RRKM rate always gives an upperbound because of "recrossing trajectories" which contribute not to 
the molecular dissociation but to the outgoing flux at the transition state. In the variational transition state theory, ${ }^{29,30}$ the position of the transition state is determined so as to minimize this harmful recrossing effect. In general, however, it has been shown by the classical mechanical analysis that the harmful effect of "premature recrossing trajectories" is unavoidable by any choice of the transition state and always makes the RRKM theory overestimate the rate. ${ }^{31,32}$ The premature recrossing trajectory corresponds to the direct collision which do not access the resonance states. In short, the direct collision, which has nothing to do with molecular predissociation, is counted in the RRKM rate and makes it an overestimate of the rate of predissociation. In the context of the overlapping resonance, the direct and resonant processes are indistinguishable at the critical overlap, where the harmful effect of direct process disappears. Consequently, the RRKM rate gives the correct predissociation rate at the critical overlap. From the scattering theoretical point of view, the RRKM rate is the maximum decay rate of resonance states allowed under the conservation of flux, and the maximum is attained at the critical overlap.

\section{COMPETITION BETWEEN IVR AND DISSOCIATION}

In the conventional understanding, the RRKM regime means the complete loss of state selectivity. ${ }^{1}$ In other words, the decay rates of many different resonance states should all become equal to the RRKM rate in this regime. The result of the random model, however, has shown that state-dependence of resonance widths always remains as fluctuation even when the average decay rate agrees with the RRKM rate. It is intuitively believed, in general, that due to rapid and complete IVR the state selectivity is completely lost in the RRKM regime. This means that the time needed for IVR to be completed should be much shorter than the dissociation lifetime. In the classical mechanical sense, trajectories should explore the whole energy shell of phase space before dissociation. We begin with a simple estimation of the time scales of the exploration of phase space and the molecular dissociation.

The time needed for the complete IVR is given by the so called recurrence time $\tau_{\mathrm{r}} \approx h \rho$. In the classical mechanical sense, this gives the time scale needed for a given trajectory to finish the exploration of phase space. ${ }^{33}$ On the other hand, the time scale of dissociation $\tau_{\mathrm{d}}$ is given by $\tau_{\mathrm{d}}=\hbar /\langle\Gamma\rangle$. With use of eq. (5) the ratio $\tau_{\mathrm{d}} / \tau_{\mathrm{r}}$ is obtained as

$$
\frac{\tau_{\mathrm{d}}}{\tau_{\mathrm{r}}} \approx \frac{1}{\langle\Gamma\rangle \rho}=\frac{\pi}{2 K} \frac{\left(1+\rho / \rho_{\mathrm{c}}\right)^{2}}{\rho / \rho_{\mathrm{c}}} .
$$

This ratio becomes minimum at $\rho=\rho_{\mathrm{c}}$, where $\tau_{\mathrm{d}} / \tau_{\mathrm{r}}=2 \pi / K<1$. This leads to $\tau_{\mathrm{d}}<$ $\tau_{\mathrm{r}}$ except for the dissociation very close to the threshold. At the critical overlap, $\rho=\rho_{\mathrm{c}}$, which corresponds to the RRKM regime in the sense that the average decay rate coincides with the RRKM rate, the dissociation takes place faster than the time scale of the recurrence. In other words, dissociation takes place before the trajectories finish the exploration of phase space. This has been pointed out also by Remacle 
and Levine. ${ }^{33}$ In order to attain $\tau_{\mathrm{d}} \gg \tau_{\mathrm{r}}$, i.e., the condition for the exploration of phase space to be completed before dissociation, we need either $\rho \gg \rho_{\mathrm{c}}$ or $\rho<<\rho_{\mathrm{c}}$, both of which lead to $k_{\mathrm{d}} \ll k_{\mathrm{RRKM}}$. The conventional picture of complete randomization before dissociation under the RRKM regime does not seem to be realized.

The competition between IVR and dissociation can be directly inspected by the auto- and mutual-correlation functions of non-stationary wave functions. The autocorrelation function of the non-stationary state $\left|\Phi_{\mathrm{i}}\right\rangle$ is defined as

$$
\begin{aligned}
\Xi_{\mathrm{ii}}(t) & =\left\langle\Phi_{\mathrm{i}}\left|e^{-i H t t h}\right| \Phi_{\mathrm{i}}\right\rangle \\
& =\sum_{n^{\prime}} \sum_{n} \sum_{v}\left\langle\Phi_{\mathrm{i}} \mid n^{\prime}\right\rangle\left\langle n^{\prime} \mid v\right\rangle e^{-i E_{v} t / \hbar} e^{-\Gamma_{v} t / 2 h}\langle v \mid n\rangle\left\langle n \mid \Phi_{\mathrm{i}}\right\rangle,
\end{aligned}
$$

This auto-correlation function is connected with the absorption spectrum $I(E)$ given in eq. (6) through the Fourier transform as ${ }^{24,25}$

$$
I(E) \propto \int_{-\infty}^{\infty} d t e^{-E t / \hbar} \Xi_{\mathrm{ii}}(t),
$$

where $\left|\Phi_{\mathrm{i}}\right\rangle$ is the vibrational wave function of the initial state in the absorption process. The manifold of resonance states is the final state of the absorption transition. Needless to say, the auto-correlation function can be obtained from the observed absorption spectrum through the inverse Fourier transform. The fine structure of the spectrum is reflected in the long time behavior of the auto-correlation function, while the global profile of the spectrum governs the short time behavior. The auto-correlation function shows how the system leaves the initial state and returns to it. On the other hand, the mutual-correlation function is given by

$$
\begin{aligned}
\Xi_{\mathrm{ii}}(t) & =\left\langle\Phi_{\mathrm{f}}\left|e^{-i H t h \mid}\right| \Phi_{\mathrm{i}}\right\rangle \\
& =\sum_{n^{\prime}} \sum_{n} \sum_{v}\left\langle\Phi_{\mathrm{f}} \mid n^{\prime}\right\rangle\left\langle n^{\prime} \mid v\right\rangle e^{-i E_{v} t h h^{-\Gamma_{v} t / 2 \hbar}}\langle v \mid n\rangle\left\langle n \mid \Phi_{\mathrm{i}}\right\rangle,
\end{aligned}
$$

and has a connection with the Raman excitation profile $\sigma_{\text {fi }}(E)$ also through the Fourier transform as $^{34-37}$

$$
\sigma_{\mathrm{fi}}(E) \propto\left|\int_{-\infty}^{\infty} d t e^{-i E t / \hbar} \Xi_{\mathrm{fi}}(t)\right|^{2},
$$

where $i$ and $f$ represent the initial and final states of the Raman scattering process. In this case, the manifold of resonance states represents the virtual intermediate states of the Raman scattering. The mutual-correlation function describes how the system visits the states different from the initial state. The IVR causes wild oscillation of both the auto- and mutual-correlation functions.

Based on the random model, the auto- and mutual-correlation functions are numerically calculated. The Hamiltonian given in eq. (3) is generated by Gaussian random numbers in the same manner as mentioned in section 3. The Franck-Condon factors $\left\langle\Phi_{\mathrm{i}} \mid n\right\rangle$ and $\left\langle\Phi_{\mathrm{f}} \mid n\right\rangle$ are also generated by random numbers so that the orthogonality 


$$
\sum_{n}\left\langle\Phi_{i} \mid n\right\rangle\left\langle n \mid \Phi_{\mathrm{f}}\right\rangle=0
$$

is retained.

The results are shown in figs. 4 and 5. In figure 4, the auto-correlation functions in the cases of isolated resonance, critical overlap and overlapping resonance are displayed. The case of overlapping resonance exhibits a typical behavior of autocorrelation function of non-integrable systems. It first decays very rapidly and shows small oscillations until the recurrence time $\tau_{\mathrm{r}} \approx \mathrm{h} \rho$, where a big peak appears. This peak is a typical recurrence, which indicates that the systems returns to the initial
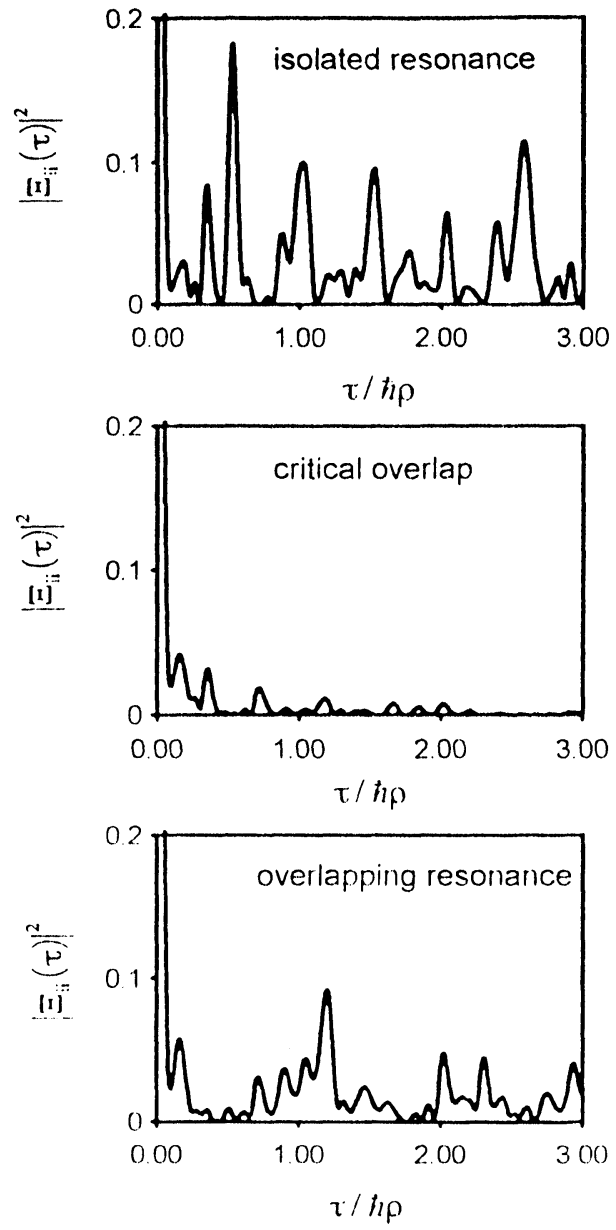

Figure 4: Auto-correlation functions obtained in the random model. The parameters are the same as those in fig. 3. The top, middle, and bottom panels correspond to the regimes of isolated resonance, critical overlap, and overlapping resonance, respectively. The correlation decays most rapidly in the regime of critical overlap. 
state after completing the exploration of phase space (or the space of quantum states). The suppression of the oscillations in $0<\tau<\hbar \rho$ is connected with the level repulsion. In the overlapping resonance, the level repulsion occurrs due to the mixing of the zero-th order resonance states via the continua. (Even in such case, the average density of state is given by $\rho=N / w$ in the random model.) The nearest neighbor level spacing distribution of the resonance states in the random model is found to be the type of Wigner and clearly shows the level repulsion in the case of overlapping resonance. On the other hand, the auto-correlation function in the isolated resonance regime shows many large amplitude oscillations. This is a typical behavior of that of integrable systems. This can be explained by the fact that there is no substantial mixing among the zero-th order resonance states in this regime and that the level spacing distribution is Poisson which characterizes integrable systems. Even in integrable systems, oscillation of the auto-correlation function, i.e., IVR, occurrs because the initial state $\left|\Phi_{i}\right\rangle$ is non-stationary state. The calculated auto-correlation function shown in fig. 4 exhibits many oscillations lasting beyond the recurrence time $\tau_{\mathrm{r}} \approx \mathrm{h} \rho$. This suggests that the complete IVR is achieved before dissociation. Lastly, the auto-correlation function in the critical overlap regime exhibits a somewhat different behavior. It decays rapidly and much less oscillations can be seen than in the regimes of overlapping resonance and isolated resonance. At the recurrence time, the auto-correlation is substantially damped. This suggests that dissociation takes place before the completion of the exploration of phase space. Figure 5 exhibits the mutual-correlation functions in the three cases. Again the case of the critical overlap shows the most quick damping of the oscillation. The physical implication is the same as in the case of the auto-correlation function: The chance of the intramolecular vibrational energy migration is minimum at the critical overlap, where the average decay rate agrees with the RRKM rate. The early stage of IVR is seen, of course, even in the critical overlap in the similar way as in the other cases: The auto-correlation function shows the first big drop and then several oscillations, and the mutual-correlation function exhibits several peaks. These phenomena characterize the early stage of IVR. However, the loss of big recurrence at the recurrence time in the case of critical overlap clearly shows that the dissociation takes place before the complete exploration of the phase space. This confirms the discussion below eq. (7). The agreement between the average decay rate and the RRKM rate at the critical overlap does not necessarily have a direct connection with the rapid and complete IVR at least within the present model.

\section{CONCLUDING REMARKS}

In the sense that the average decay rate agrees with the RRKM rate, the critical overlap of resonance can be regarded as the RRKM regime. In the scattering theoretical point of view, however, there can be found no evidence indicating that the rapid and complete IVR leads to the RRKM rate. Auto- and mutual-correlation functions suggest that insufficient time is left for IVR to be completed before dissociation particularly in the critical overlap regime, i.e., the RRKM regime. Although this 

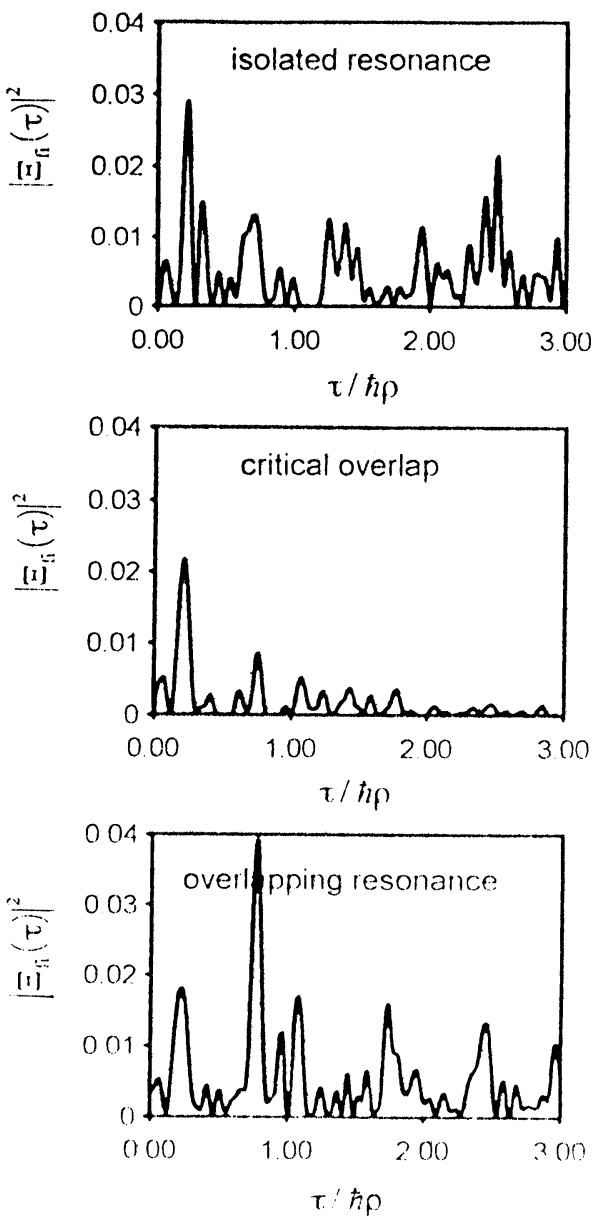

Figure 5: Mutual-correlation functions generaged in the random model. The parameters are the same as those in fig. 4. The top, middle, and bottom panels correspond to the regimes of isolated resonance, critical overlap, and overlapping resonance, respectively. Again the correlation decays most rapidly in the case of critical overlap.

observation is not parallel to the conventional belief that the rapid IVR causes the RRKM behavior, it does not contradict the existing classical mechanical analysis concerning the condition in which the RRKM rate formula is valid: The validity of the RRKM rate is attained when the contribution from the harmful recrossing trajectories, i.e., direct processes, is negligible. The existence of recrossing trajectories, however, has no direct connection with the IVR, i.e., the randomization among resonance states. The condition for the RRKM rate to be valid is attained at the critical overlap, where the resonance states are so strongly mixed with continua that the resonant processes cannot be distinguished from the direct processes. In other words, the strong mixing between the resonances and the continua leads to the RRKM regime. 


\section{Acknowledgement}

The present work was supported in part by United States-Japan Cooperative Research Program implemented by JSPS and NSF. This work is also partly supported by a Grant in Aid for Scientific Research in Priority Area "Theory of Chemical Reactions" from the Ministry of Education, Science, and Culture of Japan. The numerical computations were carried out at the computer center of the Institute for Molecular Science.

\section{References}

1. R. D. Levine and R. B. Bernstein, Molecular Reaction Dynamics and Chemical Reactivity (Oxford University Press, 1987).

2. H. Eyring, J. Chem. Phys. 3, 107 (1935).

3. E. Wigner, J. Chem. Phys. 5, 720 (1937).

4. J. C. Light, J. Chem. Phys. 40, 3221 (1964).

5. for example see S. Wiggins, Chaotic Transport in Dynamical Systems, (Springer Verlag, New York, 1992).

6. O. K. Rice, J. Phys. Chem. 65, 1588 (1961).

7. R. A. Marcus, J. Chem. Phys. 20, 359 (1952).

8. W. F. Polik, D. R. Guyer and C. B. Moore, J. Chem. Phys. 92, 3453 (1990).

9. W. F. Polik, D. R. Guyer, C. B. Moore and W. H. Miller, J. Chem. Phys. 92, 3471 (1990).

10 J. Miyawaki, K. Yamanouchi and S. Tsuchiya, J. Chem. Phys. 99, 254 (1993).

11. C. E. Porter and R. G. Thomas, Phys. Rev. 104, 483 (1956).

12. R. D. Levine, Ber. Bunsenges. Phys. Chem. 92, 222 (1988).

13. W. H. Miller, R. Hernandez, C. B. Moore and W. F. Polik, J. Chem. Phys. 93, 5657 (1990).

14. M. L. Mehta, Random Matrices (Academic Press, New York, 1967).

15. F. H. Mies and M. Krauss, J. Chem. Phys. 45, 4455 (1966).

16. K. Someda, H. Nakamura and F. H. Mies, Prog. Theor. Phys. Supplement in press.

17. K. Someda, H.Nakamura and F. H. Mies, Chem. Phys. 186 (1994) in press.

18. H. Feshbach, Annals of Phys. 5, 357 (1958).

19. H. Feshbach, Annals of Phys. 19, 287 (1962).

20 N. F. Mott and H. S. W. Massey, The Theory of Atomic Collisions (3rd ed.), (Oxford University Press, 1965).

21. U. Fano, Phys. Rev. 124, 1866 (1961).

22. F. H. Mies, Phys. Rev. 175, 164 (1968).

23. F. H. Mies and P. S. Julienne, J. Chem. Phys. 80, 2526 (1984).

24. E. J. Heller, J. Chem. Phys. 68, 2066 (1978).

25. E. J. Heller, J. Chem. Phys. 68, 3891 (1978).

26. W. H. Miller, J. Chem. Phys. 61, 1823 (1974).

27. P. Pechukas, Ann. Rev. Phys. Chem. 32, 159 (1981).

28. K. G. Kay, J. Chem. Phys. 65, 3813 (1976).

29. D. G. Truhler and B. C. Garrett, Acc. Chem. Res. 13, 440 (1980).

30 D. G. Truhler and B. C. Garrett, Ann. Rev. Phys. Chem. 35, 159 (1984).

31. M. J. Davis and S. K. Gray, J. Chem. Phys. 84, 5389 (1986).

32. S. K. Gray, S. A. Rice and M. J. Davis, J. Phys. Chem. 90, 3470 (1986).

33. F. Remacle and R. D. Levine, J. Phys. Chem. 95, 7124 (1991).

34. E. J. Heller, Acc. Chem. Res. 14, 368 (1981).

35. S. Y. Lee and E. J. Heller, J. Chem. Phys. 71, 4777 (1979).

36. E. J. Heller, R. L. Sundberg and D. J. Tannor J. Phys. Chem. 86, 1822 (1982).

37. F. Remacle, R. D. Levine and J. L. Kinsey, Chem. Phys. Lett. 205, 267 (1993). 